\title{
Periodic orbits for space-based reflectors in the circular restricted three-body problem
}

\author{
F. J. T. Salazar ${ }^{1}$ - C. R. McInnes ${ }^{2}$ - O. C. Winter ${ }^{1}$
}

Received: 29 April 2016/ Revised: 29 August 2016 / Accepted: 17 October 2016 /

Published online: 19 November 2016

CC Springer Science+Business Media Dordrecht 2016

\begin{abstract}
The use of space-based orbital reflectors to increase the total insolation of the Earth has been considered with potential applications in night-side illumination, electric power generation and climate engineering. Previous studies have demonstrated that families of displaced Earth-centered and artificial halo orbits may be generated using continuous propulsion, e.g. solar sails. In this work, a three-body analysis is performed by using the circular restricted three body problem, such that, the space mirror attitude reflects sunlight in the direction of Earth's center, increasing the total insolation. Using the Lindstedt-Poincare and differential corrector methods, a family of halo orbits at artificial Sun-Earth $\mathrm{L}_{2}$ points are found. It is shown that the third order approximation does not yield real solutions after the reflector acceleration exceeds $0.245 \mathrm{~mm} \mathrm{~s}^{-2}$, i.e. the analytical expressions for the in- and out-of-plane amplitudes yield imaginary values. Thus, a larger solar reflector acceleration is required to obtain periodic orbits closer to the Earth. Derived using a two-body approach and applying the differential corrector method, a family of displaced periodic orbits close to the Earth are therefore found, with a solar reflector acceleration of $2.686 \mathrm{~mm} \mathrm{~s}^{-2}$.
\end{abstract}

Keywords Halo orbit - Artificial libration point - Displaced orbit - Space reflectors · Earth's climate system · Three-body problem

$凶$ F. J. T. Salazar

e7940@ hotmail.com

C. R. McInnes

Colin.McInnes@glasgow.ac.uk

O. C. Winter

ocwinter@gmail.com

1 UNESP-Grupo de Dinâmica Orbital e Planetologia, Guaratinguetá, SP 12516-410, Brazil

2 School of Engineering, University of Glasgow, Glasgow G12 8QQ, UK 


\section{Introduction}

The global mean Earth's surface temperature depends mainly on the net amount of radiation arriving on the Earth's surface (McGuffie and Henderson-Sellers 2005). Space-based geoengineering schemes propose to alter the amount of incoming sunlight at the Earth's surface, changing the average global temperature with minimal consequences for the ecosystem and expected favorable results (McInnes 2010a). Supposing that a period of cooling similar to the 'little ice ace' occurred from the sixteenth and nineteenth centuries (Le Roy Ladurie 1971; Free and Robock 1999) was coming, or future supervolcanic eruption took place, e.g. Toba mega-eruption (Zielinski et al. 1996), which would alter the reflectivity of the Earth and cool the climate. Then, in the twenty-first century, a colder weather would greatly impact agriculture, economics, and energy demand. Therefore, it is interesting to speculate on future engineering plans to avoid such climate cooling.

The use of orbiting mirrors to reflect sunlight to the Earth has been the field of study for different applications, e.g. increasing the length of daylights for electric power generation or lighting up the darks days in the winter (Glaser 1968; Oberth 1972; Ehricke 1979; Canady and Allen 1982; Leary 1993; Frass et al. 2013), or the so-called Mars climate engineering (Oberg 1981; McKay et al. 1991; Fogg 1995; Zubrin and McKay 1997; McInnes 2002, 2010b), due to the great quantity of energy leverage delivered rapidly by the mirrors (Maunter and Parks 1990). Thus, large-scale solar reflectors seem to be a fascinating instrument to prevent the occurrence of cooling events that have taken place in the past.

In this work, in order to redirect solar radiation to increase the amount of sunlight on Earth's surface, two suitable locations have been considered in the circular restricted three body problem (CRTBP) to deploy a solar reflector. The first location is at an artificial Sun-Earth $\mathrm{L}_{2}$ point, which can be generated from a continuous acceleration, e.g. solar sail or electric low thrust propulsion system (McInnes et al. 1994; McInnes 1999; Baig and McInnes 2009; Morimoto et al. 2007). By considering the solar radiation pressure on a perfectly flat reflecting space mirror, it is shown that the required equilibrium condition, so that sunlight is reflected in the direction of the Earth's center, generates artificial $L_{2}$ points everywhere on the $x$-axis from the natural $\mathrm{L}_{2}$ point towards the Earth's center. The first goal of this work is to find periodic orbits about an artificial $\mathrm{L}_{2}$ point, such that the mirror attitude is oriented to guarantee that the sunlight is reflected in the direction of Earth's center. Analytical and numerical $\mathrm{L}_{2}$ halo orbits for a solar reflector are obtained using the Lindstedt-Poincaré and differential corrector methods (Thurman and Worfolk 1996), respectively. Analytical solutions are derived from a third order Taylor series expansion about artificial $\mathrm{L}_{2}$ point, while the differential corrector method uses as first guess the third order solution (Szebehely 1967; McInnes 1999; Baoyin and McInnes 2006; Baig and McInnes 2009; Salazar and Winter 2016). The analytical results yield imaginary solutions after the reflector acceleration exceeds $0.245 \mathrm{~mm} \mathrm{~s}^{-2}$, i.e. close to the natural $\mathrm{L}_{2}$ equilibrium point. Thus, a larger solar reflector acceleration is required to obtain periodic orbits closer to the Earth.

Displaced Earth-centered orbits are an attractive option to deploy space reflectors and increase the reflector acceleration. Since solar radiation force greatly affects the reflector orbits about Earth (Salazar et al. 2016), a family of non-Keplerian polar orbits for solar reflectors can be generated managing the reflector orientation, so that the solar radiation pressure applies a force on the mirror surface, dislocating the polar orbit from the Earth's center to behind it along the Sun-Earth line (McInnes and Simmons 1992). A two-body analysis, i.e. Earth-reflector approximation, generates periodic orbits relative to an inertial frame, which can be introduced as initial guesses in the differential corrector method, so that 
displaced Earth-centered orbits can then be obtained for the Sun-Earth-reflector three-body problem.

\section{Equations of motion}

The motion of a solar space reflector in the Sun-Earth system can be approximated by the equations of the CRTBP (Szebehely 1967). In this approach, there exist five natural equilibrium points, known as Lagrangian points. However, for the Sun-Earth-reflector threebody problem, the solar reflector attitude may be oriented so that new artificial equilibrium solutions may be generated (McInnes et al. 1994).

Assuming the distance between the Sun and Earth, the sum of the masses of them and gravitational constant equal to unity, the vector equation of motion for an idealised, perfectly reflecting solar reflector can then be written with respect to a synodic rotating frame of reference $(x, y, z)$ with unit angular velocity vector $\omega$ and origin at their common center-ofmass, as (McInnes et al. 1994)

$$
\ddot{\mathbf{r}}+2 \omega \times \dot{\mathbf{r}}=\nabla V+\mathbf{a},
$$

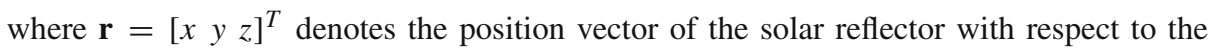
center of mass, as shown in Fig. 1, and the solar radiation acceleration a and the effective potential function $V$ are defined by

$$
\begin{aligned}
\mathbf{a} & =\beta \frac{1-\mu}{r_{1}^{2}}\left(\hat{\mathbf{r}}_{1} \cdot \mathbf{n}\right)^{2} \mathbf{n}, \\
V & =\left(\frac{1-\mu}{r_{1}}+\frac{\mu}{r_{2}}\right)+\frac{1}{2}(\boldsymbol{\omega} \times \mathbf{r}) \cdot(\boldsymbol{\omega} \times \mathbf{r}),
\end{aligned}
$$

where the mass ratio $\mu=3.04036 \times 10^{-6}$ (Richardson 1980), $\mathbf{r}_{1}=\left[\begin{array}{lll}x+\mu & y & z\end{array}\right]^{T}$ and $\mathbf{r}_{2}=\left[\begin{array}{lll}x-(1-\mu) & y & z\end{array}\right]^{T}$ denote the position vectors of the space reflector with respect to the Sun and Earth, respectively. In addition, $\hat{\mathbf{r}}_{1}$ is directed along the Sun-line, $\mathbf{n}$ is the reflector attitude unit normal vector, and $\beta$ is the reflector lightness number.

Equilibrium solutions require that $\ddot{\mathbf{r}}=\dot{\mathbf{r}}=0$. It then can be shown that the equilibrium condition implies that the required reflector attitude in the synodic rotating frame for new artificial equilibrium points is given by (McInnes et al. 1994)

Fig. 1 Earth-Sun-reflector circular restricted three-body problem, in order that sunlight is reflected in the direction of the Earth's center. $\mathrm{L}_{1}$ and $\mathrm{L}_{2}$ represent the natural collinear equilibrium solutions and the reflector attitude angle is denoted by $\alpha$ (not to scale)

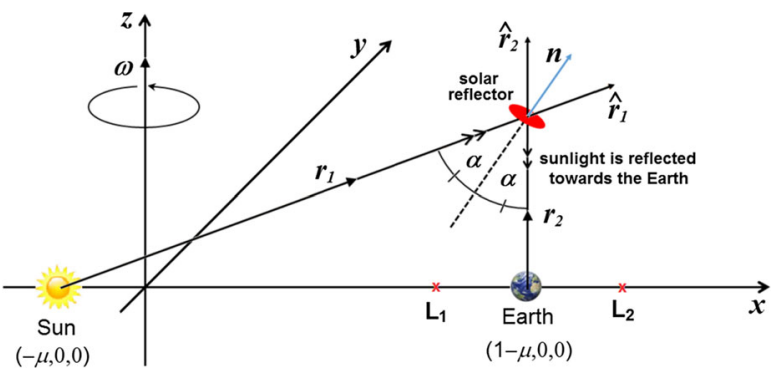




$$
\mathbf{n}=-\frac{\nabla V}{|\nabla V|}
$$

Denoting by $\alpha$ the angle between the incident sunlight vector $\hat{\mathbf{r}}_{1}$ and the normal vector $\mathbf{n}$, we know that, according to the law of reflection, the angle of incidence must be equal to the angle of reflection. Therefore, if the mirror is reflecting sunlight towards the Earth's center, then the law of reflection requires

$$
\mathbf{n}=\frac{\hat{\mathbf{r}}_{1}+\hat{\mathbf{r}}_{2}}{\left|\hat{\mathbf{r}}_{1}+\hat{\mathbf{r}}_{2}\right|},
$$

where $\hat{\mathbf{r}}_{2}$ is directed along the Earth-line, as shown in Fig. 1. Since we need to guarantee that the sunlight is reflected in the direction of Earth's center, we will only focus on equilibrium solutions that satisfy simultaneously Eqs. (4) and (5). These regions can be easily found by taking the scalar product $\mathbf{n}_{1} \cdot \mathbf{n}_{2}=\frac{\nabla V \cdot\left(\hat{\mathbf{r}}_{1}+\hat{\mathbf{r}}_{2}\right)}{|\nabla V|\left|\hat{\mathbf{r}}_{1}+\hat{\mathbf{r}}_{2}\right|}$, where $\mathbf{n}_{1}=-\frac{\nabla V}{|\nabla V|}$ and $\mathbf{n}_{2}=\frac{\left(\hat{\mathbf{r}}_{1}+\hat{\mathbf{r}}_{2}\right)}{\left|\hat{\mathbf{r}}_{1}+\hat{\mathbf{r}}_{2}\right|}$. From the scalar product, we can define the angle $\theta=\cos ^{-1}\left(\mathbf{n}_{1} \cdot \mathbf{n}_{2}\right)$. Thus, the surfaces defined by $\theta=0^{\circ}$ where $\mathbf{n}_{1}=\mathbf{n}_{2}$, correspond to equilibrium solutions where the required reflector attitude is satisfied.

Evaluating this constraint, it is found that the family of equilibrium points, that redirect sunlight in the direction of the Earth's center, lies on $x$-axis from the Lagrangian point $\mathrm{L}_{2}$ to the Earth's center as shown in Fig. 2. Additionally, the definition of solar radiation acceleration, Eq. (2), and the orientation of artificial equilibrium points, Eq. (4), imply that the $x$-coordinate of the artificial $\mathrm{L}_{2}$ points depends explicitly on the lightness number $\beta$. In the next sections, analytical and numerical periodic orbits (i.e. halo orbits) about these new $\mathrm{L}_{2}$ points will be obtained.

\section{Reflector Sun-Earth $L_{2}$ point halo orbits}

\subsection{Third-order expansion}

To find periodic orbits about the new collinear equilibrium points, the equations of motion near an equilibrium position $\mathbf{r}_{0}=\left[\begin{array}{lll}x & 0 & 0\end{array}\right]^{T}$, with $1-\mu<x<1.0101$ (natural $\mathrm{L}_{2}$ point location), will be approximated as shown in Fig. 2. Although shadows effects are important considerations (McInnes 1999; Pavlak 2011), the eclipses will not be included in this investigation. The scope of this analysis is restricted to understanding the existence of periodic orbits about new collinear points for solar reflectors in the Sun-Earth system. Thus, defining the vector force $\mathbf{F}=\nabla V+\mathbf{a}$, such that, the reflector normal vector $\mathbf{n}$ obeys the constraint Eq. (5), the definition of solar radiation pressure yields an expression of the form

Fig. 2 Space reflector in a periodic halo orbit about an artificial equilibrium point beyond $\mathrm{L}_{2}$, so that sunlight is redirected towards the Earth. The artificial point is denoted by $\mathrm{L}_{2}^{*}$ (not to scale)

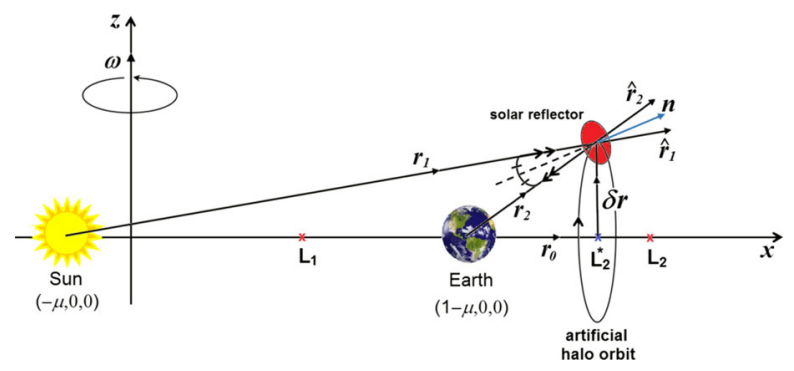




$$
\begin{aligned}
\mathbf{a} & =\beta \frac{1-\mu}{r_{1}^{2}}\left(\hat{\mathbf{r}}_{1} \cdot\left(\frac{\hat{\mathbf{r}}_{1}+\hat{\mathbf{r}}_{2}}{\left|\hat{\mathbf{r}}_{1}+\hat{\mathbf{r}}_{2}\right|}\right)\right)^{2} \frac{\hat{\mathbf{r}}_{1}+\hat{\mathbf{r}}_{2}}{\left|\hat{\mathbf{r}}_{1}+\hat{\mathbf{r}}_{2}\right|}, \\
& =\beta \frac{1-\mu}{r_{1}^{2}}\left(\frac{1+\hat{\mathbf{r}}_{1} \cdot \hat{\mathbf{r}}_{2}}{\left|\hat{\mathbf{r}}_{1}+\hat{\mathbf{r}}_{2}\right|}\right)^{2} \frac{\hat{\mathbf{r}}_{1}+\hat{\mathbf{r}}_{2}}{\left|\hat{\mathbf{r}}_{1}+\hat{\mathbf{r}}_{2}\right|}
\end{aligned}
$$

A Taylor series expansion of $\mathbf{F}$ to third-order about $\mathbf{r}_{0}$ is then found by substituting the vector position $\mathbf{r}=\mathbf{r}_{0}+\delta \mathbf{r}$ into Eq. (1) (Edwards 1973):

$$
\delta \ddot{\mathbf{r}}+2 \omega \times \delta \dot{\mathbf{r}}=\left[\frac{\mathrm{d} \mathbf{F}}{\mathrm{d} \mathbf{r}}\right]_{\mathbf{r}_{0}} \delta \mathbf{r}+\frac{1}{2 !}\left[\frac{\mathrm{d}^{2} \mathbf{F}}{\mathrm{d}^{2}}\right]_{\mathbf{r}_{0}} \delta \mathbf{r}^{2}+\frac{1}{3 !}\left[\frac{\mathrm{d}^{3} \mathbf{F}}{\mathrm{d} \mathbf{r}^{3}}\right]_{\mathbf{r}_{0}} \delta \mathbf{r}^{3}+O\left(\delta \mathbf{r}^{4}\right),
$$

where $\delta \mathbf{r}=\left[\begin{array}{lll}\delta x & \delta y & \delta z\end{array}\right]^{T}$, and $\left[\frac{\mathrm{d} \mathbf{F}}{\mathrm{d} \mathbf{r}}\right]_{\mathbf{r}_{0}},\left[\frac{\mathrm{d}^{2} \mathbf{F}}{\mathrm{d} \mathbf{r}^{2}}\right]_{\mathbf{r}_{0}},\left[\frac{\mathrm{d}^{3} \mathbf{F}}{\mathrm{d} \mathbf{r}^{3}}\right]_{\mathbf{r}_{0}}$ are the first, second and third derivative matrices of vector force $\mathbf{F}=\left[\begin{array}{lll}F^{x} & F^{y} & F^{z}\end{array}\right]^{T}$ about $\mathbf{r}_{0}$, respectively. Additionally, the second and third order terms for $\delta x, \delta y, \delta z$ are included in $\delta \mathbf{r}^{2}$ and $\delta \mathbf{r}^{3}$, respectively.

The scalar form of Eq. (7) is given by

$$
\begin{aligned}
\delta \ddot{x}-2 \delta \dot{y} & =A \delta x+C \delta x^{2}+D\left(\delta y^{2}+\delta z^{2}\right)+F \delta x^{3}+G \delta x\left(\delta y^{2}+\delta z^{2}\right), \\
\delta \ddot{y}+2 \delta \dot{x} & =(1+B) \delta y+E \delta x \delta y+H \delta y^{3}+I \delta y\left(\delta x^{2}+\delta z^{2}\right), \\
\delta \ddot{z} & =B \delta z+E \delta x \delta z+H \delta z^{3}+\delta z\left(I \delta x^{2}+J \delta y^{2}\right),
\end{aligned}
$$

where $A=\left.F_{x}^{x}\right|_{\mathbf{r}_{0}}, B=\left.F_{z}^{z}\right|_{\mathbf{r}_{0}}, C=\frac{\left.F_{x x}^{x}\right|_{\mathbf{r}_{0}}}{2}, D=\frac{\left.F_{y y}^{x}\right|_{\mathbf{r}_{0}}}{2}, E=\left.F_{x y}^{y}\right|_{\mathbf{r}_{0}}, F=\frac{\left.F_{x x x}^{x}\right|_{\mathbf{r}_{0}}}{6}$, $G=\left.\frac{F_{x y z}^{x} \mid}{2}\right|_{\mathbf{r}_{0}}, H=\frac{\left.F_{y y y}^{y}\right|_{\mathbf{r}_{0}}}{6}, I=\frac{\left.F_{x x y}^{y}\right|_{\mathbf{r}_{0}}}{2}$, and $J=\frac{\left.F_{x z z}^{y}\right|_{\mathbf{r}_{0}}}{2}$. Thus, the constant terms in Eq. (8) depends explicitly on the $x$-coordinate of artificial $\mathrm{L}_{2}$ point.

\subsection{Third-order solution}

In this section, in order to to obtain periodic solutions to the equations of motion, Eq. (8), the Lindstedt-Poincaré method will be used. Firstly, we will remove the nonlinear terms in Eq. (8), so that the problem is reduced to a linear analysis about $\mathbf{r}_{0}$. It is then found that the linear equations of motion can be written as $\delta \dot{\mathbf{X}}=\mathbf{M} \delta \mathbf{X}$, where $\delta \mathbf{X}=[\delta \mathbf{r} \delta \dot{\mathbf{r}}]^{T}$ and the matrix $\mathbf{M}$ is given by

$$
\mathbf{M}=\left[\begin{array}{cc}
\mathbf{0} & \mathbf{I} \\
\mathcal{U} & \Omega
\end{array}\right]
$$

where $\mathbf{I}$ is the identity matrix, and the components of the matrices $\mathcal{U}$ and $\boldsymbol{\Omega}$ are given by

$$
\mathcal{U}=\left[\begin{array}{ccc}
A & 0 & 0 \\
0 & 1+B & 0 \\
0 & 0 & B
\end{array}\right], \boldsymbol{\Omega}=\left[\begin{array}{ccc}
0 & 2 & 0 \\
-2 & 0 & 0 \\
0 & 0 & 0
\end{array}\right]
$$

Note that the $z$-component is decoupled from $x, y$, and that the out-of-plane motion corresponds to a periodic oscillation with angular frequency $\sqrt{-B}$. On the other hand, it can be shown that the characteristic polynomial for the $x, y$ linearized dynamics has two pairs of purely imaginary eigenvalues $\pm i \lambda$ and one pair of real eigenvalues $\pm s$, corresponding to a center and saddle points, respectively (Farquhar et al. 1977; Richardson 1980; Morimoto et al. 2007; Baig and McInnes 2009). Since we are only interested in bounded solutions for 
$\delta x$ and $\delta y$, it is easy to show that the linear problem defined by Eq. (9) possesses a bounded solution

$$
\begin{aligned}
& \delta x=X \cos \lambda t, \\
& \delta y=\kappa X \sin \lambda t, \\
& \delta z=\eta X \cos \sqrt{-B} t,
\end{aligned}
$$

where the in-plane frequency and the ratio of $y$ - and $x$-axis amplitude are given by

$$
\begin{aligned}
\lambda & =\sqrt{-\frac{A+B-3}{2}+\sqrt{\left(\frac{A+B-3}{2}\right)^{2}-A(1+B)},} \\
\kappa & =-\frac{\lambda^{2}+A}{2 \lambda},
\end{aligned}
$$

and $\eta=\frac{Z}{X}$ is an free parameter.

Since the linear solution is not, in general, periodic, then nonlinearities should be included to find periodic solutions about $\mathbf{r}_{0}$ (Farquhar et al. 1977; Richardson 1980; Farquhar et al. 1980).

The Lindstedt-Poincaré method assumes that if the nonlinear terms are small, then a periodic solution of the nonlinear system Eq. (8) is essentially a perturbation of a periodic solution of the linear system Eq. (9) (Drazin 1992), which can be determined introducing a frequency correction, i.e. a new parameter $\tau=w t$, where $w=1+\varepsilon w_{1}+\varepsilon^{2} w_{2}$. Then the original problem becomes

$$
\begin{aligned}
w^{2} \delta x^{\prime \prime}-2 w \delta y^{\prime} & =A \delta x+C \delta x^{2}+D\left(\delta y^{2}+\delta z^{2}\right)+F \delta x^{3}+G \delta x\left(\delta y^{2}+\delta z^{2}\right), \\
w^{2} \delta y^{\prime \prime}+2 w \delta x^{\prime} & =(1+B) \delta y+E \delta x \delta y+H \delta y^{3}+I \delta y\left(\delta x^{2}+\delta z^{2}\right), \\
w^{2} \delta z^{\prime \prime}+\lambda^{2} \delta z & =E \delta x \delta z+H \delta z^{3}+\delta z\left(I \delta x^{2}+J \delta y^{2}\right)+\Delta \delta z,
\end{aligned}
$$

where $\Delta=\lambda^{2}+B$, and $\delta x^{\prime}, \delta x^{\prime \prime}$ denote the first and second derivatives with respect to $\tau$ (similarly for $\delta y$ and $\delta z$ coordinates).

To solve the nonlinear system Eq. (14), it will be supposed solutions of the form

$$
\begin{aligned}
& \delta x=\varepsilon \delta x_{1}+\varepsilon^{2} \delta x_{2}+\varepsilon^{3} \delta x_{3}, \\
& \delta y=\varepsilon \delta y_{1}+\varepsilon^{2} \delta y_{2}+\varepsilon^{3} \delta y_{3}, \\
& \delta z=\varepsilon \delta z_{1}+\varepsilon^{2} \delta z_{2}+\varepsilon^{3} \delta z_{3},
\end{aligned}
$$

and that $\Delta=O\left(\varepsilon^{2}\right)$ (Thurman and Worfolk 1996). Substituting these expressions into the equations of motion Eq. (14), and grouping components of the same order in $\varepsilon$, then system of equations of first, second and third order, as well as their periodic solutions, can be obtained as shown in Eqs. (28) through (33) (see "Appendix 1" section). The secular terms of the systems of equations of second and third order, are cancelled through the following constraints

$$
\begin{aligned}
w_{1} & =0, \\
w_{2} & =s_{1} X^{2}+s_{2} Z^{2}, \\
l_{1} X^{2}+l_{2} Z^{2}+\Delta & =0 .
\end{aligned}
$$

The expressions for $s_{i}, l_{i}$ are listed in "Appendix 3" section. The $\varepsilon$ term is removed from all the equations changing the scale of variables by the function $X \rightarrow \frac{X}{\varepsilon}$ and $Z \rightarrow \frac{Z}{\varepsilon}$. 
Fig. 3 In-plane and out-of-plane frequencies for the linear approximation and $x$-coordinate of artificial $\mathrm{L}_{2}$ point versus lightness number

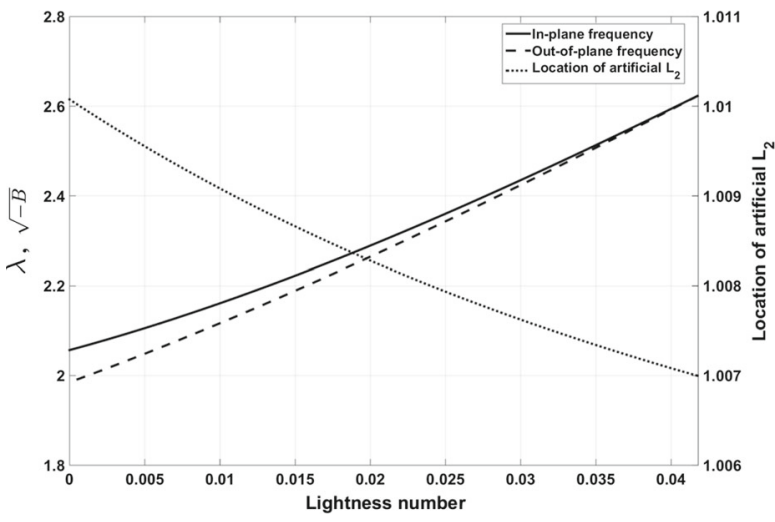

Combining the periodic solutions of the first, second and third order equations, the complete periodic solution for the third-order approximation Eq. (7) is given by

$$
\begin{aligned}
& \delta x(t)=X \cos \tau_{1}+P_{20}+P_{2} \cos 2 \tau_{1}+P_{3} \cos 3 \tau_{1}, \\
& \delta y(t)=\kappa X \sin \tau_{1}+Q_{2} \sin 2 \tau_{1}+Q_{3} \sin 3 \tau_{1}, \\
& \delta z(t)=\eta X \sin \tau_{1}+M_{2}\left(3-\cos 2 \tau_{1}\right)+M_{3} \cos 3 \tau_{1},
\end{aligned}
$$

where $\tau_{1}=\lambda w t$, and $\lambda, \kappa, \eta$ come from the linear approximation, Eq. (11). The expression for the coefficients $P_{i}, Q_{i}, M_{i}$ are given in "Appendix 2" section. This solution corresponds to a halo orbit about the new artificial $\mathrm{L}_{2}$ point, whose period $T$ is equal to $\frac{2 \pi}{\lambda w}$, where $w=1+s_{1} X^{2}+s_{2} Z^{2}$, and the minimum in-plane amplitude $X$ can be computed from Eq. (18) by setting $Z=0$, i.e. $X \geq \sqrt{-\frac{\Delta}{l_{1}}}$. Counterclockwise motion corresponds to positive values of $\eta$.

Figure 3 shows the $x$-coordinate of the artificial $\mathrm{L}_{2}$ point, as well as the in-plane and outof-plane frequencies of the linearized system Eq. (9), as a function of the lightness number $\beta$. The location of the natural $\mathrm{L}_{2}$ point corresponds to $\beta=0$, and the difference between the in-plane and out-of-plane frequencies is quite small, or approximately, a relative difference of $3.7 \%$. For $\beta \neq 0$, the relative difference decreases as the lightness number increases, and it approaches zero when $\beta=0.042$. This fact implies that the second order frequency correction $w_{2}$ is small when $\beta=0$ and it approaches zero at $\beta=0.042$, as shown below.

Figures 4 and 5 show the $x$-direction amplitude $X$ and the period for the artificial halo orbits, respectively, with the $z$-direction amplitude set as $\eta=0,1,2,3$. For $\beta=0$ and $\eta \approx 1$, an amplitude $X$ of approximately $200,000 \mathrm{~km}$ and an orbit period of 180 days correspond to a classical halo orbit about $\mathrm{L}_{2}$ point. As can be seen in Figs. 4 and 5 , the amplitude $X$ and orbit period are decreasing functions when $0 \leq \beta \leq 0.042$, obtaining an amplitude of approximately $1400 \mathrm{~km}$ (about one-quarter the Earth's radius) and an orbit period of 140 days at $\beta=0.042$. Note the amplitude $X$ decreases much faster than orbit period does. Therefore, the third order approximation and the reflectivity constraint, Eq. (5), produce periodic solutions that converge quickly to the equilibrium points when $\beta$ increases. For $\beta>0.042$ the expression $\frac{\Delta}{l_{1}+l_{2} \eta^{2}}$ in Eq. (18) is strictly positive for $\eta=0,1,2,3$. Therefore, imaginary solutions are found for the third-order approximation given by Eq. (19) when $\beta>0.042$ and $|\eta| \leq 3$.

Finally, Fig. 6 shows the second order frequency correction $w_{2}$ and $\lambda$ as functions of the lightness number. As noted above, $w_{2}$ is very small at $\beta=0$ and it approaches zero quickly. 
Fig. $4 x$-direction amplitude $X$ for halo orbits at artificial $\mathrm{L}_{2}$ near to the natural $\mathrm{L}_{2}$ point versus lightness number, with various $Z$

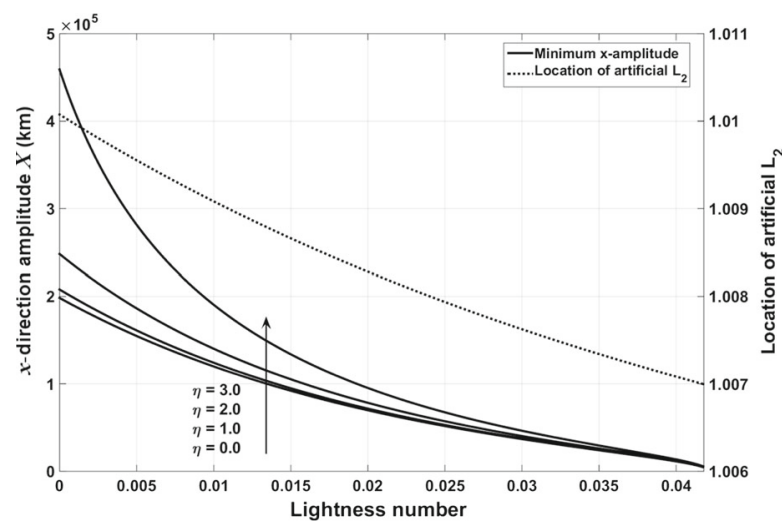

Fig. 5 Orbit period of halo orbits at artificial $\mathrm{L}_{2}$ near to natural $\mathrm{L}_{2}$ point versus lightness number, with various $Z$

Fig. 6 Second order frequency correction $w_{2}$ and $\lambda$ versus lightness number, with various $Z$
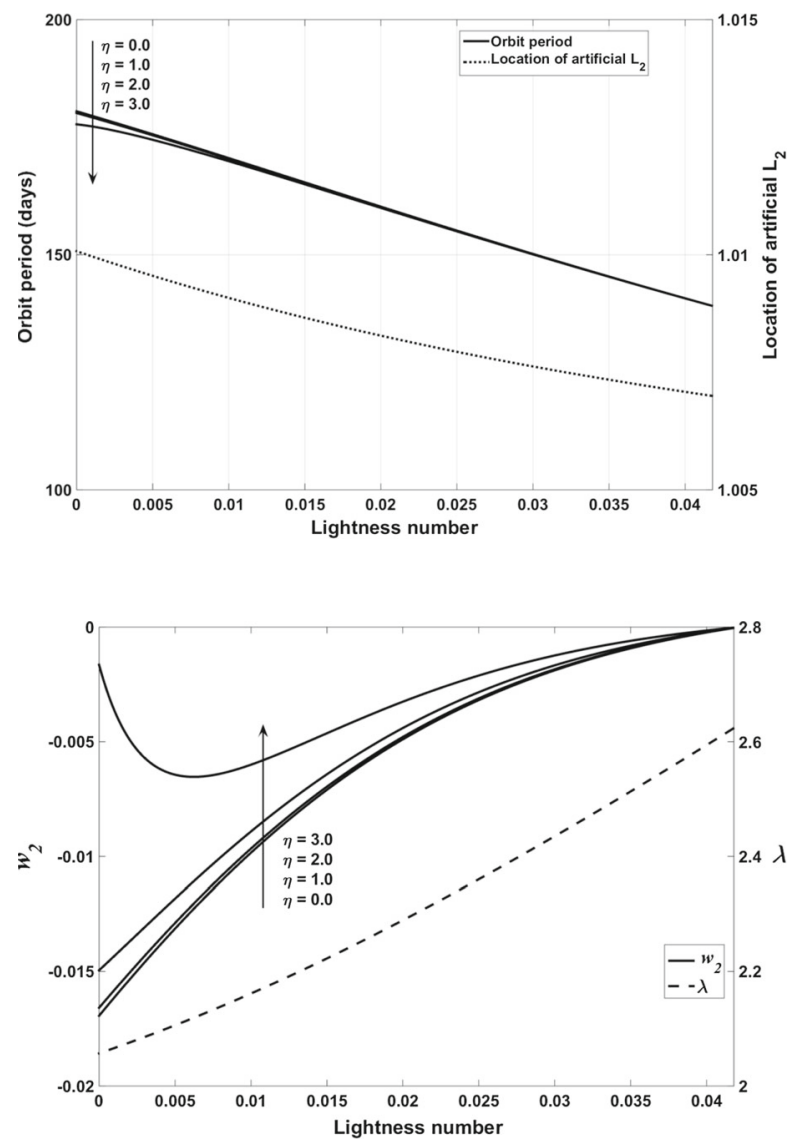

Thus, the $z$-frequency approaches $\lambda$ as $\beta$ increases. Figures 4, 5 and 6 also show that the $z$-direction amplitude practically does not affect the $x$-direction amplitude, orbit period $T$ and $w_{2}$ when $X<2 Z$. 


\section{Numerical computation of artificial Halo orbits}

As explained in Sect. 3.2, the pair of real eigenvalues $\pm s$ for the in-plane linear approximation implies that the new $\mathrm{L}_{2}$ points are unstable. Therefore, the initial conditions given by the Lindstedt-Poincaré analysis are not enough to generate periodic solutions for the CRTBP with solar perturbation. For the purpose of computing periodic orbits, the differential corrector method can be used, which is an iterative method that starts with the third order approximation as an initial guess, and after successively better approximations, new initial conditions converge to a desired final state (Koon et al. 2011).

Since halo orbits are symmetric about the $x z$-plane $(y=0)$ and that they intersect this plane perpendicularly $(\dot{x}=\dot{z}=0)$, then let $\mathbf{X}_{0}=\left[\begin{array}{llllll}x_{0} & 0 & z_{0} & 0 & \dot{y}_{0} & 0\end{array}\right]^{T}$ be an initial state given by the Lindstedt-Poincaré approximation, Eq. (19), which intersects perpendicularly the $x z$ plane. On the first return to the $x y$-plane at $t=t_{f}$, its state is $\mathbf{X}_{f}=\left[\begin{array}{llllll}x_{f} & 0 & z_{f} & \dot{x}_{f} & \dot{y}_{f} & \dot{z}_{f}\end{array}\right]^{T}$. Thus, a periodic solution exists if $\dot{x}_{f}=\dot{z}_{f}=0$. Since $\dot{x}_{f}, \dot{z}_{f}$ may not be zero, the three non-zero initial conditions $\left(x_{0}, z_{0}\right.$, and $\left.\dot{y}_{0}\right)$ can be altered slightly with the purpose of driving these velocities to zero.

Let $\Delta \mathbf{X}(t)$ denote a slight variation relative to the reference solution $\overline{\mathbf{X}}(t)$ (known) corresponding to $\mathbf{X}_{0}$. Supposing that the initial and final displacements are small enough, they are linearly related by the following expression (Thurman and Worfolk 1996)

$$
\Delta \mathbf{X}\left(t_{f}+\Delta t\right)=\boldsymbol{\Phi}\left(t_{f}, t_{0}\right) \Delta \mathbf{X}\left(t_{0}\right)+\dot{\overline{\mathbf{X}}}\left(t_{f}\right) \Delta t,
$$

where $\boldsymbol{\Phi}\left(t_{f}, t_{0}\right)$ is the state transition matrix from time $t_{0}$ to $t_{f}$, evaluated along the reference solution $\overline{\mathbf{X}}(t)$ (Koon et al. 2011). If $\mathbf{X}_{0}+\Delta \mathbf{X}\left(t_{0}\right)$ belongs to a halo orbit, then the variation $\Delta \mathbf{X}(t)$ when the trajectory crosses the $x z$-plane is given by

$$
\Delta \mathbf{X}\left(t_{f}+\Delta t\right)=\left[\begin{array}{lllll}
x_{f}^{*}-x_{f} & 0 & z_{f}^{*}-z_{f}-\dot{x}_{f} & \dot{y}_{f}^{*}-\dot{y}_{f}-\dot{z}_{f}
\end{array}\right]^{T},
$$

where $\mathbf{X}_{f}^{*}=\left[\begin{array}{llllll}x_{f}^{*} & 0 & z_{f}^{*} & 0 & \dot{y}_{f}^{*} & 0\end{array}\right]^{T}$ denotes the halo orbit intersection with the $x z$-plane at $t=t_{f}+\Delta t$, as the desired final state (unknown). Thus, the correction terms $\left(\Delta x_{0}, \Delta z_{0}\right.$, $\Delta \dot{y}_{0}$, and $\Delta t$ ) can be determined from Eq. (20). For simplicity, it will be assumed $\Delta z_{0}=0$, so that the variations $\Delta x_{0}, \Delta \dot{y}_{0}$ and $\Delta t$ can be easily computed inverting a $3 \times 3$ matrix as follows

$$
\left[\begin{array}{c}
\Delta x_{0} \\
\Delta \dot{y}_{0} \\
\Delta t
\end{array}\right]=\left[\begin{array}{lll}
\phi_{21} & \phi_{25} & \dot{y}_{f} \\
\phi_{41} & \phi_{45} & \ddot{x}_{f} \\
\phi_{61} & \phi_{65} & \ddot{z}_{f}
\end{array}\right]^{-1}\left[\begin{array}{c}
0 \\
-\dot{x}_{f} \\
-\dot{z}_{f}
\end{array}\right],
$$

where $\phi_{i j}$ are elements of the matrix $\boldsymbol{\Phi}\left(t_{t}, t_{0}\right)$. The new initial conditions $\mathbf{X}_{0}+\Delta \mathbf{X}_{0}$ are then used to begin a second iteration, until $\dot{x}_{f}=\dot{z}_{f}=0$ (or within some acceptable tolerance).

Figures 7,8 and 9 show a family of halo orbits obtained by the differential corrector method for $\beta=0,0.02,0.042$ and $z$-direction amplitude setting $\eta=0,1,2$. Figure 7 shows halo orbits around the natural $\mathrm{L}_{2}$ point. In Figs. 8 and 9, the solar reflector, placed on the artificial halo orbit, redirects sunlight towards the Earth's center.

The reflector areal density with $\beta=0.042$ is given by $\sigma=\frac{1.53}{\beta}=36.42 \mathrm{~g} \mathrm{~m}^{-2}$ (McInnes 1999). This value corresponds to a space reflector acceleration of $0.245 \mathrm{~mm} \mathrm{~s}^{-2}$. Therefore, the reflector acceleration cannot exceed $0.245 \mathrm{~mm} \mathrm{~s}^{-2}$ for the artificial halo orbits found in this work. Similar results are obtained by Baoyin and McInnes (2006) and Baig and McInnes (2009) for halo orbits about artificial $\mathrm{L}_{1}$ and $\mathrm{L}_{2}$ points in the Sun-Earth-spacecraft CRTBP using solar sail and low-thrust propulsion, respectively, such that, the reflector normal vector 

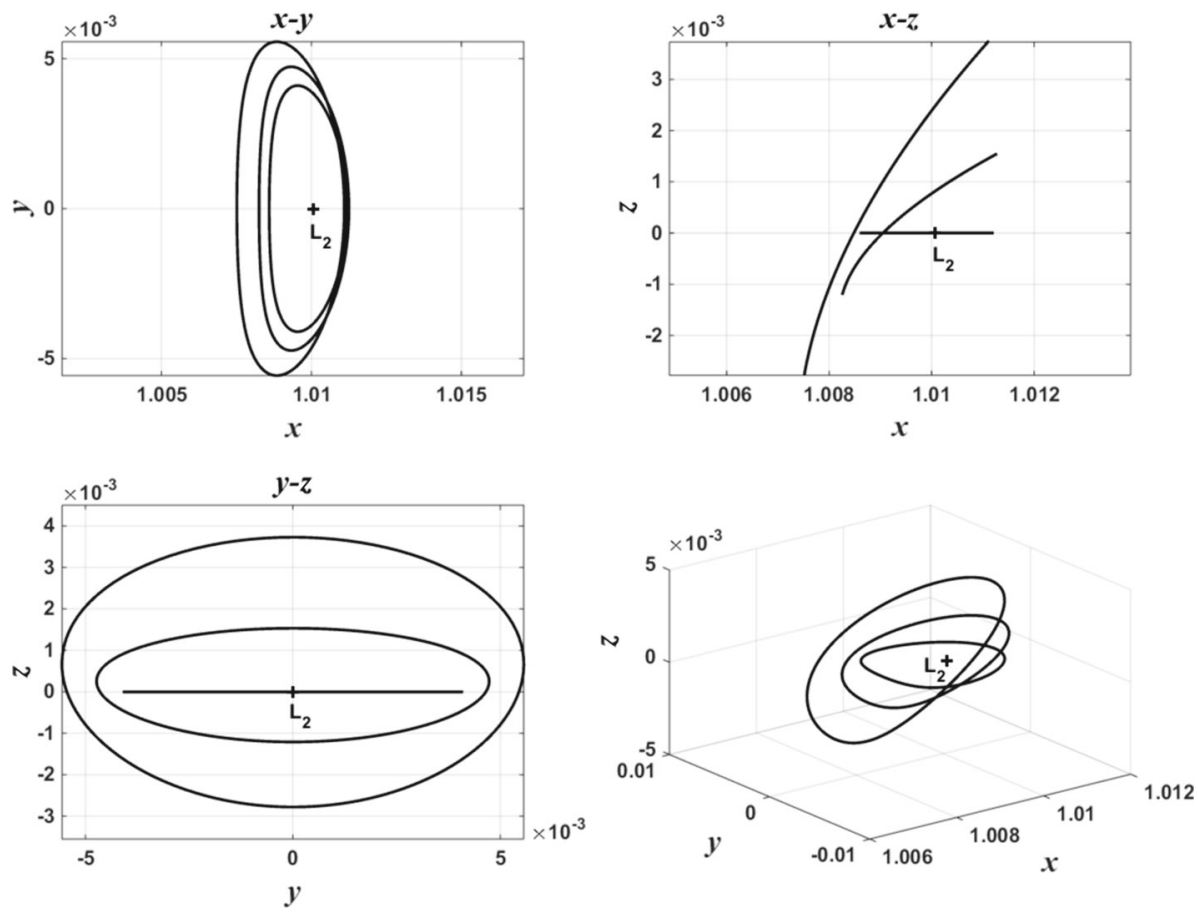

Fig. 7 Natural halo orbits around $\mathrm{L}_{2}$ point with $\beta=0$ and $\eta=0,1,2$
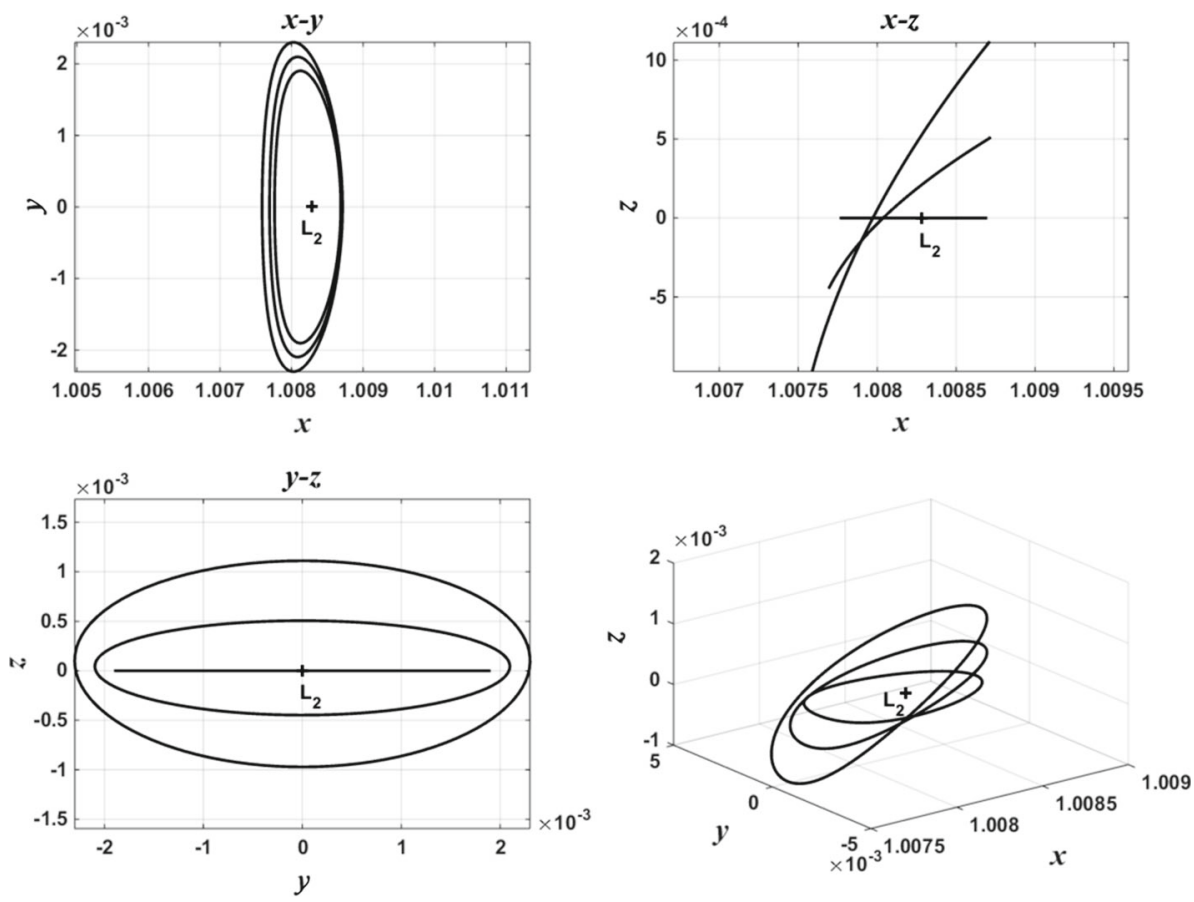

Fig. 8 Artificial halo orbits around new $\mathrm{L}_{2}$ point with $\beta=0.02$ and $\eta=0,1,2$ 

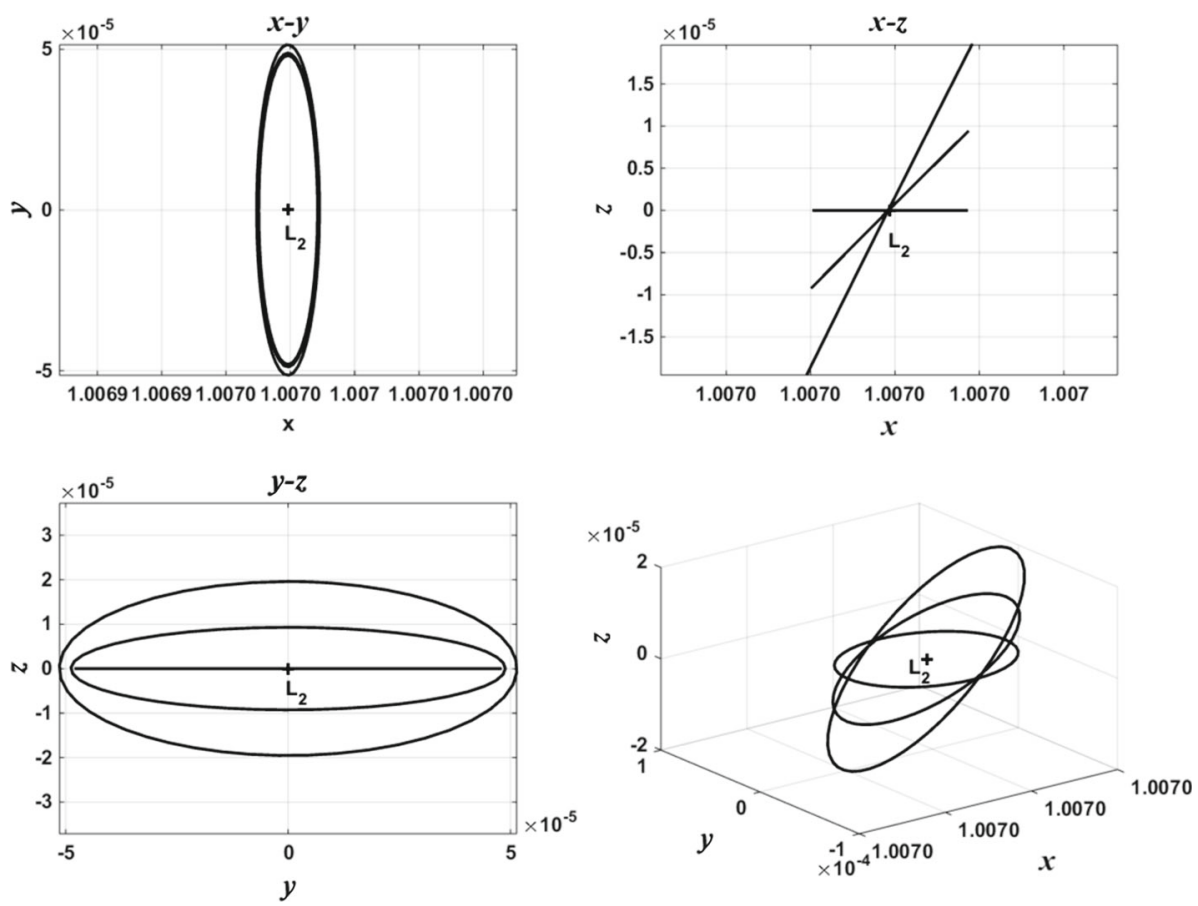

Fig. 9 Artificial halo orbits around new $\mathrm{L}_{2}$ point with $\beta=0.042$ and $\eta=0,1,2$

is directed along the Sun-spacecraft line (reflector attitude angle $\alpha=0^{\circ}$ ) or along the SunEarth line (reflector attitude vector $\mathbf{n}=\left[\begin{array}{lll}1 & 0 & 0\end{array}\right]^{T}$ ). However, in this paper, both the angle $\alpha$ and vector $\mathbf{n}$ are changing about the orbit to satisfy the reflectivity constraint, Eq. (5).

Large scale space-based geo-engineering proposals require minimizing the mass per unit area $\sigma$ (McInnes 2010a), so that a larger reflector acceleration is required. The third order approximation, used in this work, produces imaginary values for the $x$-direction amplitude $X$ when $\beta>0.042$. It is therefore necessary to use another scheme that permits increasing the lightness number (i.e. reflector acceleration).

\section{Displaced Earth-centered orbits}

Displaced polar orbits have been studied for different applications, e.g. solar sails, orbiting reflectors to increase the amount of sunlight (McInnes and Simmons 1992; Dankowicz 1994; McInnes 1999, 2010b; Bewick et al. 2011; Salazar et al. 2016). These are basically polar circular orbits, displaced behind Earth along the Sun-Earth line due to the solar radiation force exerted on the space mirror surface, as shown in Fig. 10 (McInnes and Simmons 1992).

Although the existence of this kind of orbits has been demonstrated in the two-body problem, and more accurate models like Hill's approximation have been studied (Bookless and McInnes 2006), it is possible to consider them as an initial guess in the differential corrector method described previously, such that bounded solutions can be obtained in the Sun-Earth-reflector CRTBP.

In order to obtain an initial state, a perfect reflector placed at $\mathbf{r}_{p}$ will be assumed. The rotation of the reflector will be about the $X$-axis of an inertial coordinate system $X Y Z$, with 
Fig. 10 Space mirror on an Earth-centered displaced orbit

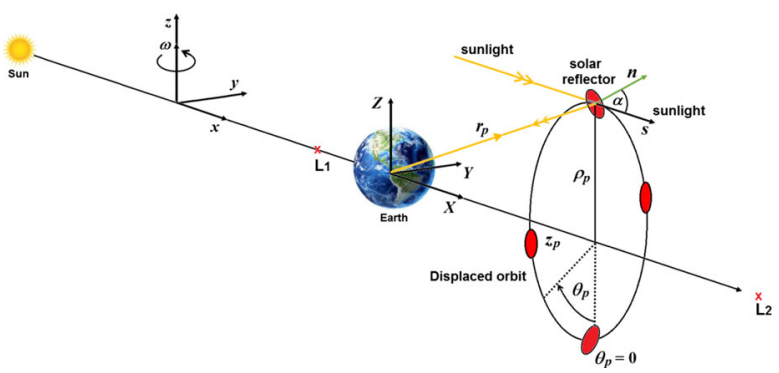

the origin of the frame at the Earth's center, as shown in Fig. 10. Similarly, the angle between the reflector normal unit vector $\mathbf{n}$ and the Sun-line will be defined by $\alpha$, and the characteristic reflector acceleration will be denoted by $a$. In this two-body approximation, it is assumed that the Sun-line is given by the unit vector $\mathbf{s}=\left[\begin{array}{lll}1 & 0 & 0\end{array}\right]^{T}$, i.e. the motion of the Sun around the Earth is not accounted for.

In cylindrical polar coordinates $\left(\rho_{p}, \theta_{p}, z_{p}\right)$ the equations of motion for a perfect space mirror are (McInnes 2010b)

$$
\begin{array}{r}
\ddot{\rho}_{p}-\rho_{p} \dot{\theta}_{p}^{2}+\frac{\rho_{p}}{r_{p}}\left(\frac{\mu_{E}}{r_{p}^{2}}\right)-a \cos ^{2} \alpha \sin \alpha=0, \\
\rho_{p} \ddot{\theta}_{p}+2 \dot{\rho}_{p} \dot{\theta}_{p}=0, \\
\ddot{z}_{p}+\frac{z_{p}}{r_{p}}\left(\frac{\mu_{E}}{r_{p}^{2}}\right)-a \cos \alpha^{3}=0,
\end{array}
$$

where $\mu_{E}=398600.440 \mathrm{~km}^{3} \mathrm{~s}^{-2}$ is the Earth gravitational constant and $r_{p}=\sqrt{\rho_{p}^{2}+z_{p}^{2}}$.

A circular displaced orbit must satisfy $\ddot{\rho}_{p}=\ddot{\theta}_{p}=\ddot{z}_{p}=0$ and $\dot{\rho}_{p}=\dot{z}_{p}=0$. On the other hand, in order to guarantee that the space mirror reflects sunlight towards the Earth's center, the geometry of the incident ray (see Figs. 1 and 10) yields an expression for the attitude angle $\alpha$

$$
\tan \alpha=\tan \left(\frac{1}{2} \tan ^{-1}\left(\frac{\rho_{p}}{z_{p}}\right)\right) .
$$

Therefore, if this orientation is fixed previously, the required orbital angular velocity and characteristic acceleration for an Earth-centered displaced orbit can be found from Eq. (23) to be

$$
\begin{gathered}
w_{p}=\tilde{w}_{p}\left(1-\left(\frac{z}{p}\right) \tan \alpha\right)^{1 / 2}, \\
a=z_{p} \tilde{w}_{p}^{2}\left(1+\tan \alpha^{2}\right)^{3 / 2},
\end{gathered}
$$

where $w_{p}=\dot{\theta}_{p}$ and $\tilde{w}_{p}^{2}=\frac{\mu_{E}}{r_{p}^{3}}$.

From Eq. (26), fixed values for $a$ can be chosen, so that contour lines of constant acceleration are produced in the $\rho_{p}-z_{p}$ plane, as shown in Fig. 11, where it can be seen that a larger reflector acceleration is possible for orbits close to the Earth. Additionally, a linear stability analysis has shown that the boundary $\rho_{p}=4 z_{p}$ (dashed line in Fig. 11) separates the $\rho_{p}-z_{p}$ plane into stable $\left(\rho_{p}>4 z_{p}\right)$ and unstable regions (McInnes 2010b). Therefore, circular polar 
Fig. 11 Contour lines of constant reflector acceleration $a$ $\left(\mathrm{mm} \mathrm{s}^{-2}\right)$, the dashed line $\left(\rho_{p}=4 z_{p}\right)$ separates the $\rho_{p}-z_{p}$ plane into stable $\left(\rho_{p}>4 z_{p}\right)$ and unstable regions

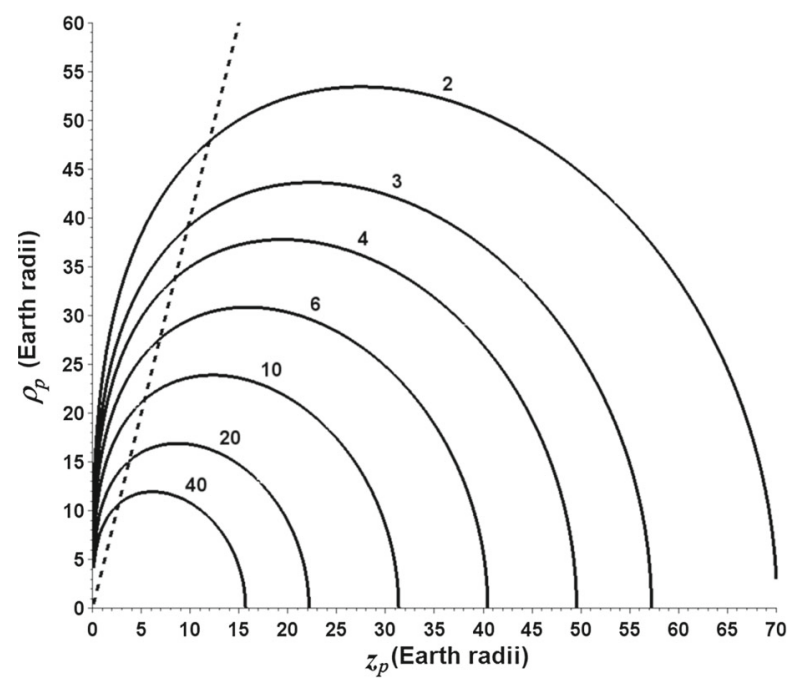

orbits inside the stable region, will be defined as an initial state in the differential corrector method.

Fixing a radius $\rho_{p}$ and a displacement $z_{p}$, so that the space reflector intersects perpendicularly the $x z$-plane (see Fig. 10), the corresponding coordinates with respect to the inertial frame $X Y Z$ are given by $\left(z_{p}, 0,-\rho_{p}, 0,-\rho_{p} w_{p}, 0\right)$. Thus, the coordinates with respect to the synodic rotating frame, corresponding to the initial state $\mathbf{X}_{0}=\left[\begin{array}{llllll}x_{0} & 0 & z_{0} & 0 & \dot{y}_{0} & 0\end{array}\right]^{T}$, are obtained as

$$
\begin{aligned}
& x_{0}=z_{p}+1-\mu, \\
& z_{0}=-\rho_{p}, \\
& \dot{y}_{0}=-\left(z_{p}+\rho_{p} w_{p}\right),
\end{aligned}
$$

where $\rho_{p}, z_{p}$, and $w_{p}$ have been made nondimensional. Thus, the differential corrector is applied by iterating from the initial guess $\mathbf{X}_{0}$.

Figure 12 shows a family of 1-year displaced orbits in the CRTBP, obtained by a numerical integration of Eq. (1), and using as initial states circular polar orbits that belong to the linear stable region shown in Fig. 11. The Earth is illustrated by an Earth-centered blue circle that has the same radius of the Earth in normalized units. Figure 12 shows four displaced orbits with initial radius $\rho_{p}=\left(4 z_{p}+1\right)$ Earth radii, where the displacement $z_{p}=2.5,5,7.5,10$ Earth radii, and the reflector acceleration $a=35.83,10.1,4.68,2.69 \mathrm{~mm} \mathrm{~s}^{-2}$, respectively. Thus, the differential corrector method permits to find a new set of displaced orbits in the Sun-Earth-reflector CRTBP. Previous works have only obtained this kind of orbits in the twobody problem, assuming fixed solar acceleration vector directed along the Sun-line, as was described previously (Dankowicz 1994; McInnes 1999, 2010b; Bewick et al. 2011; Salazar et al. 2016).

Although linear stable circular polar orbits could be generated with any displacement $z_{p}$ in the two-body scenario, as shown in Fig. 11, in three-body scenario, orbits with large displacements are found to be strongly perturbed by solar gravitational force. Additionally, the characteristic acceleration $a$ decreases as displacement $z_{p}$ increases for $\rho_{p}>4 z_{p}$, as shown in Fig. 11. Despite that the solar gravitational perturbations will affect the dynamics 

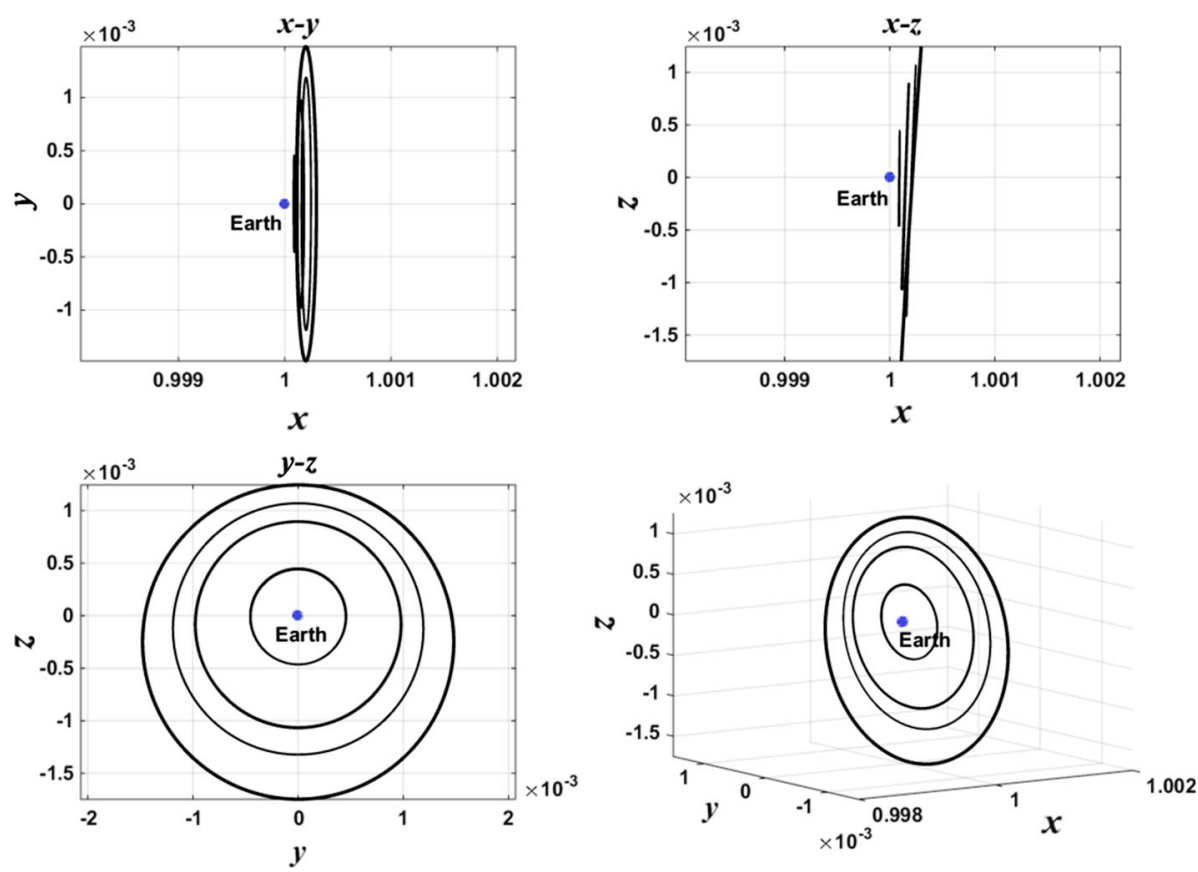

Fig. 12 Displaced orbits with $\rho_{0}=\left(4 z_{p}+1\right)$ Earth radii, where the displacement $z_{p}=2.5,5,7.5,10$ Earth radii, and the reflector acceleration $a=35.83,10.1,4.68,2.69 \mathrm{~mm} \mathrm{~s}^{-2}$, respectively. Earth is illustrated by an Earth-centered blue circle that has the same radius of the Earth in normalized units

of the solar reflector, it is possible to increase the acceleration, even for large displacements. The cost for that is the loss of stability. For example, Fig. 13 shows a classical unbounded orbit for $\rho_{p}<4 z_{p}$ in the CRTBP, with initial radius $\rho_{p}=41$ Earth radii, initial displacement $z_{p}=40$ Earth radii, and reflector acceleration $a=2.671 \mathrm{~mm} \mathrm{~s}^{-2}$. Note that the value obtained for the acceleration is similar to the value computed for the displaced orbit presented in Fig. 12. In this way, previous studies using the two-body problem and Hill's equations as an approximation to the CRTBP, have designed control techniques to avoid escape from the nominal displaced orbit (McInnes 1999; Bookless and McInnes 2006).

A periodic behavior for space-based reflector orbits in the CRTBP exists then near the Earth and near the natural $\mathrm{L}_{2}$ point. However, the linear analysis in the two-body problem and the third order approximation are not enough to determine the existence of periodic orbits between these two locations. Only unbounded displaced orbits far from the Earth are found in this paper, as shown in Fig. 13.

\section{Conclusions}

Space-based solar reflectors have been considered to increase the amount of sunlight on the Earth's surface. New $\mathrm{L}_{2}$ equilibrium points were found in the Sun-Earth-reflector CRTBP, so that the reflector attitude redirected the sunlight towards the Earth. In this manner, a family of artificial halo orbits about new $\mathrm{L}_{2}$ points were obtained through a third order 

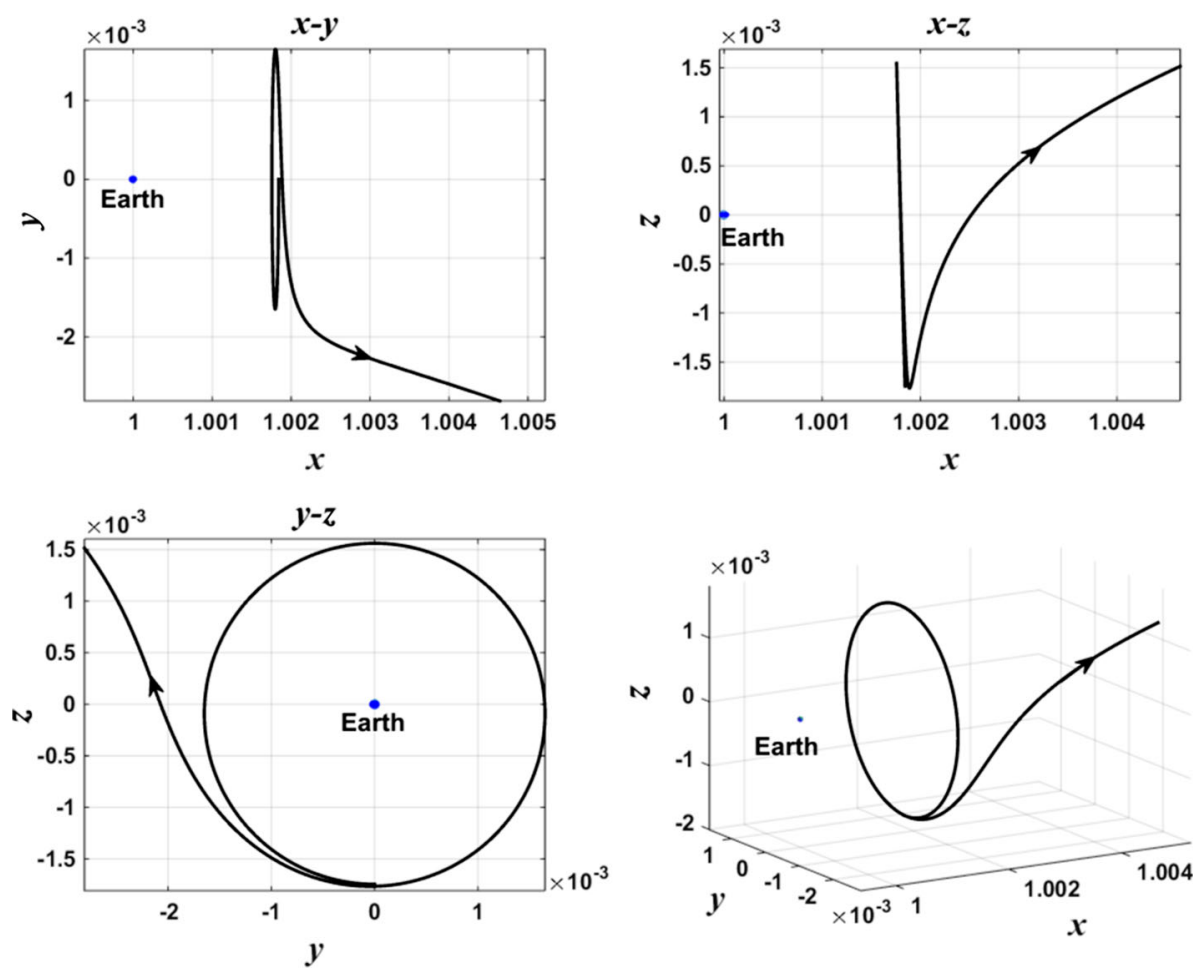

Fig. 13 Unbounded Earth-centered orbit with $\rho_{0}=41$ Earth radii, $z_{0}=40$ Earth radii, and $a=2.671 \mathrm{~mm}$ $\mathrm{s}^{-2}$. Earth is illustrated by an Earth-centered blue circle that has the same radius of the Earth in normalized units

approximation, such that the sunlight was reflected onto the Earth's center by the reflectors deployed at these locations. Due to the instability of the new equilibrium points, the third order analytical solutions were used as an initial guess in the differential corrector method, which was implemented to find numerical halo orbits. Artificial halo orbits were obtained near the natural Sun-Earth $\mathrm{L}_{2}$ point, where the reflector acceleration did not exceed 0.245 $\mathrm{mm} \mathrm{s}^{-2}$.

In order to increase the reflector acceleration, i.e. to reduce the areal density, a family of displaced Earth-centered orbits has been obtained. Considering the Earth-reflector two-body problem as an initial approximation, the required initial states have been derived as a function of the radius and displacement of the orbit, and used in the differential corrector method to obtain bounded solutions in the Sun-Earth CRTBP. Thus, displaced orbits relatively close to the Earth were found. As a result, the reflector acceleration was greatly increased with respect to the space mirrors placed on the artificial halo orbit. Additionally, unbounded displaced orbits with larger displacements from the Earth were also found.

Acknowledgements The authors would like to thank the financial support of the FAPESP (São Paulo Research Foundation, Brazil), Grants 2011/08171-3, 2013/03233-6, 2015/00559-3 and the CNPq (National Council for Scientific and Technological Development, Brazil), and the technical support of University of Glasgow. C.R.M was support by a Royal Society Wolfson Research Merit Award. Finally, the authors would like to thank the reviewers for the comments and suggestions. 


\section{Appendix 1: Lindstedt-Poincaré method}

First order equations and their periodic solutions

$$
\begin{aligned}
\delta x_{1}^{\prime \prime}-2 \delta y_{1}^{\prime}-A \delta x_{1} & =0 \\
\delta y_{1}^{\prime \prime}+2 \delta x_{1}^{\prime}-(1+B) \delta y_{1} & =0 \\
\delta z_{1}^{\prime \prime}+\lambda^{2} \delta z_{1} & =0 \\
\delta x_{1}(\tau) & =X \cos \lambda \tau \\
\delta y_{1}(\tau) & =\kappa X \sin \lambda \tau \\
\delta z_{1}(\tau) & =\eta X \cos \lambda \tau
\end{aligned}
$$

Second order equations and their periodic solutions

$$
\begin{aligned}
\delta x_{2}^{\prime \prime}-2 \delta y_{2}^{\prime}-A \delta x_{2} & =-2 w_{1} \delta x_{1}^{\prime \prime}+2 w_{1} \delta y_{1}^{\prime}+C \delta x_{1}^{2}+D\left(\delta y_{1}^{2}+\delta z_{1}^{2}\right) \\
\delta y_{2}^{\prime \prime}+2 \delta x_{2}^{\prime}-(1+B) \delta y_{2} & =-2 w_{1} \delta y_{1}^{\prime \prime}-2 w_{1} \delta x_{1}^{\prime}+E \delta x_{1} \delta y_{1} \\
\delta z_{2}^{\prime \prime}+\lambda^{2} \delta z_{2} & =-2 w_{1} \delta z_{1}^{\prime \prime}+E \delta x_{1} \delta z_{1} \\
\delta x_{2}(\tau) & =P_{20}+P_{2} \cos 2 \lambda \tau \\
\delta y_{2}(\tau) & =Q_{2} \sin 2 \lambda \tau \\
\delta z_{2}(\tau) & =M_{2}(3-\cos 2 \lambda \tau)
\end{aligned}
$$

Third order equations and their periodic solutions

$$
\begin{aligned}
\delta x_{3}^{\prime \prime}-2 \delta y_{3}^{\prime}-A \delta x_{3}= & -w_{1}^{2} \delta x_{1}^{\prime \prime}-2 w_{2} \delta x_{1}^{\prime \prime}-2 w_{1} \delta x_{2}^{\prime \prime}+2 w_{2} \delta y_{1}^{\prime}+2 w_{1} \delta y_{2}^{\prime} \\
& +2 C \delta x_{1} \delta x_{2}+2 D\left(\delta y_{1} \delta y_{2}+\delta z_{1} \delta z_{2}\right)+F \delta x_{1}^{3} \\
& +G \delta x_{1}\left(\delta y_{1}^{2}+\delta z_{1}^{2}\right) \\
\delta y_{3}^{\prime \prime}+2 \delta x_{3}^{\prime}-(1+B) \delta y_{3}= & -w_{1}^{2} \delta y_{1}^{\prime \prime}-2 w_{2} \delta y_{1}^{\prime \prime}-2 w_{1} \delta y_{2}^{\prime \prime}-2 w_{2} \delta x_{1}^{\prime}-2 w_{1} \delta x_{2}^{\prime} \\
& +E\left(\delta x_{1} \delta y_{2}+\delta x_{2} \delta y_{1}\right)+\delta y_{1}\left(I \delta x_{1}^{2}+H \delta y_{1}^{2}+J \delta z_{1}^{2}\right) \\
\delta z_{3}^{\prime \prime}+\lambda^{2} \delta z_{3}= & -w_{1}^{2} \delta z_{1}^{\prime \prime}-2 w_{2} \delta z_{1}^{\prime \prime}-2 w_{1} \delta z_{2}^{\prime \prime}+\frac{\Delta}{\varepsilon^{2}} \delta z_{1} \\
& +E\left(\delta x_{1} \delta z_{2}+\delta x_{2} \delta z_{1}\right)+\delta z_{1}\left(I \delta x_{1}^{2}+J \delta y_{1}^{2}+H \delta z_{1}^{2}\right) \\
\delta x_{3}(\tau)= & P_{3} \cos 3 \lambda \tau \\
\delta y_{3}(\tau)= & Q_{3} \sin 3 \lambda \tau \\
\delta z_{3}(\tau)= & M_{3} \cos 3 \lambda \tau
\end{aligned}
$$

\section{Appendix 2: Coefficients}

\section{First order coefficients}

$$
\lambda=\sqrt{-\frac{A+B-3}{2}+\sqrt{\left(\frac{A+B-3}{2}\right)^{2}-A(1+B)}}
$$




$$
\kappa=-\frac{\lambda^{2}+A}{2 \lambda}
$$

\section{Second order coefficients}

$$
\begin{aligned}
P_{20} & =p_{20} X^{2} \\
P_{2} & =p_{2} X^{2} \\
Q_{2} & =q_{2} X^{2} \\
M_{2} & =\eta m_{2} X^{2} \\
p_{20} & =-\frac{\left(C+D\left(\eta^{2}+\kappa^{2}\right)\right)}{2 A} \\
p_{2} & =\frac{\left(4 \lambda^{2}+B+1\right)\left(C+D\left(\eta^{2}-\kappa^{2}\right)\right)-4 \lambda \kappa E}{2 r_{2}} \\
q_{2} & =\frac{\kappa E\left(4 \lambda^{2}+A\right)-4 \lambda\left(C+D\left(\eta^{2}-\kappa^{2}\right)\right)}{2 r_{2}} \\
r_{2} & =16 \lambda^{2}-\left(4 \lambda^{2}+A\right)\left(4 \lambda^{2}+B+1\right) \\
m_{2} & =\frac{E}{6 \lambda^{2}}
\end{aligned}
$$

Third order coefficients

$$
\begin{aligned}
P_{3} & =\frac{6 \lambda q_{33}-\left(9 \lambda^{2}+B+1\right) p_{33}}{r_{3}} \\
Q_{3} & =\frac{6 \lambda p_{33}-\left(9 \lambda^{2}+A\right) q_{33}}{r_{3}} \\
M_{3} & =\frac{m_{33}}{8 \lambda^{2}} \\
p_{33} & =\left(C P_{2}-\eta D M_{2}-\kappa D Q_{2}\right) X+\frac{1}{4}\left(F+G\left(\eta^{2}-\kappa^{2}\right)\right) X^{3} \\
q_{33} & =\frac{E}{2}\left(\kappa P_{2}+Q_{2}\right) X+\frac{\kappa}{4}\left(I+\eta^{2} J-\kappa^{2} H\right) X^{3} \\
r_{3} & =\left(9 \lambda^{2}+A\right)\left(9 \lambda^{2}+B+1\right)-36 \lambda^{2} \\
m_{33} & =\frac{E}{2}\left(\eta P_{2}-M_{2}\right) X+\frac{\kappa}{4}\left(I+\eta^{2} H-\kappa^{2} J\right) X^{3}
\end{aligned}
$$

\section{Appendix 3: Coefficients for the nonsecular terms constraint}

$$
\begin{aligned}
s_{1}=\frac{1}{s} & {\left[\left(\lambda^{2}+1+B\right)\left(C\left(2 p_{20}-p_{2}\right)+\kappa q_{2} D+\frac{1}{4}\left(3 F+\kappa^{2} G\right)\right)\right.} \\
& \left.-2 \lambda\left(E\left(\kappa\left(p_{20}-\frac{1}{2} p_{2}\right)+\frac{1}{2} q_{2}\right)+\frac{\kappa}{4}\left(I+3 \kappa^{2} H\right)\right)\right] \\
s_{2}= & \frac{1}{s}\left[\left(\lambda^{2}+1+B\right)\left(7 m_{2} D+\frac{3}{4} G\right)-\frac{1}{2} \lambda \kappa J\right] \\
s= & 4 \lambda^{2}(1+\kappa \lambda)-2 \lambda(\lambda+\kappa)\left(\lambda^{2}+1+B\right)
\end{aligned}
$$




$$
\begin{aligned}
& l_{1}=\frac{E}{2}\left(5 m_{2}+2 p_{20}+p_{2}\right)+\frac{1}{4}\left(3 I+\kappa^{2} J\right)+2 s_{1} \lambda^{2} \\
& l_{2}=\frac{3}{4} H+2 s_{2} \lambda^{2}
\end{aligned}
$$

\section{References}

Baig, S., McInnes, C.R.: Artificial halo orbits for low-thrust propulsion spacecraft. Celest. Mech. Dyn. Astron. 104(4), 321-335 (2009). doi:10.1007/s10569-009-9215-4

Baoyin, H., McInnes, C.: Solar sail halo orbits at the Sun-Earth artificial L1 point. Celest. Mech. Dyn. Astron. 94(2), 155-171 (2006). doi:10.1007/s10569-005-4626-3

Bewick, R., Sanchez, J.P., McInnes, C.R.: Use of orbiting reflectors to decrease the technological challenges of surviving the lunar night. In: 62nd International Astronautical Congress, International Astronautical Federation, Cape Town, South Africa (2011)

Bookless, J., McInnes, C.: Dynamics and control of displaced periodic orbits using solar-sail propulsion. J. Guid. Control Dyn. 29(3), 527-537 (2006). doi:10.2514/1.15655

Canady, J.E., Jr., Allen, J.L., Jr: Illumination from Space with Orbiting Solar-Reflector Spacecraft, NASA TP-2065 (1982)

Dankowicz, H.: Some special orbits in the two-body problem with radiation pressure. Celest. Mech. Dyn. Astron. 58(4), 353-370 (1994). doi:10.1007/BF00692010

Drazin, P.G.: Nonlinear Systems. Cambridge University Press, Cambridge (1992)

Edwards, C.H.: Advanced Calculus of Several Variables. Academic Press, New York (1973). ISBN 978-0486-68336-2

Ehricke, K.A.: Space light: space industrial enhancement of the solar option. Acta Astronaut. 6(12), 1515-1633 (1979)

Farquhar, R.W., Muhonen, D.P., Richardson, D.L.: Mission design for a halo orbiter of the earth. J. Spacecr. Rockets 14(3), 170-177 (1977). doi:10.2514/6.1976-810

Farquhar, R.W., Muhonen, D.P., Newman, C.R., Heuberber, H.S.: Trajectories and orbital maneuvers for the first libration-point satellite. J. Guid. Control 3, 549-554 (1980). doi:10.2514/3.56034

Fogg, M.J.: Terraforming: Engineering Planetary Environments. SAE International, Warrendale (1995)

Frass, L.M., Derbes, B., Palisoc, A.: Mirrors in dawn dusk orbit for low-cost terrestrial solar electric power in the evening. In: 51st AIAA Aerospace Sciences Meeting, Grapevine, Texas (2013). doi:10.2514/6.20131191

Free, M., Robock, A.: Global warming in the context of the little ice age. J. Geophys. Res. 104(D16), 1905719070 (1999)

Glaser, P.E.: Power from the Sun: its future. Science 162, 857-861 (1968)

Koon, W.S., Lo, M.W., Marsden, J.E., Ross, S.D.: Dynamical Systems, the Three-Body Problem and Space Mission Design. Marsden Books, Wellington (2011). ISBN 978-0-615-24095-4

Le Roy Ladurie, E.: Times of feast, times of famine: a history of climate since the year 1000. Translated from the French by Barbara Bray, Doubleday, Garden City, NY (1971)

Leary, W.E.: Russians to test space mirror as giant night light for Earth-New York Times. The New York Times-Breaking News, World News \& Multimedia, The New York Times, 12 Jan 1993. Web. 11 Dec 2011. http://www.nytimes.com/1993/01/12/science/russians-to-test-space-mirror-as- giant-night-lightfor-earth.html?pagewanted=all. Accessed 17 Oct 2015

Maunter, M., Parks, K.: Space-Based Climate Control, Engineering, Construction and Operations in Space II. In: Proceedings Space 1990. American Society of Civil Engineers, Reston (1990)

McGuffie, K., Henderson-Sellers, A.: A climate modelling primer, 3rd edn. Wiley, Hoboken (2005)

McInnes, C.R.: Solar Sailing: Technology, Dynamics and Mission Applications. Springer, London (1999)

McInnes, C.R.: Non-Keplerian orbits for Mars solar reflectors. J. Br. Interplanet. Soc. 55, 74-84 (2002)

McInnes, C.R.: Space-based geoengineering: challenges and requirements. Proc. Inst. Mech. Eng. C J. Mech. Eng. Sci. 224(3), 571-580 (2010a). doi:10.1243/09544062JMES1439

McInnes, C.R.: Mars climate engineering using orbiting solar reflectors. In: Badescu, V. (ed.) Mars: Prospective Energy and Material Resources, pp. 645-659. Springer, Heidelberg (2010b)

McInnes, C.R., Simmons, J.F.L.: Halo orbits for solar sails II-geocentric case. J. Spacecr. Rockets 29(4), 472-479 (1992). doi:10.2514/3.55639

McInnes, C.R., Mcdonald, A.J.C., Simmons, J.F.L., MacDonald, E.: Solar sail parking in restricted three-body system. J. Guid. Control Dyn. 17(2), 399-406 (1994). doi:10.2514/3.21211

McKay, C., Kasting, J., Toon, O.: Making Mars habitable. Nature 352, 489-496 (1991). doi:10.1038/352489a0 
Morimoto, M., Yamakawa, H., Uesugi, K.: Artificial equlibrium points in the low-thrust restricted three-body problem. J. Guid. Control Dyn. 30(5), 1563-1567 (2007). doi:10.2514/1.26771

Oberg, J.E.: New Earths: Restructuring Earth and Other Planets. New American Library Inc, New York (1981)

Oberth, H.: Ways to Spaceflight. NASA Technical Translation, TT F-622 (1972)

Pavlak, T.A.: Modeling and Simulation of a Spacecraft Influenced by Shadowing as a Hybrid System. AAE 690 Final Project Report. 15 Dec 2011, Purdue University (2011)

Richardson, D.L.: Analytic construction of periodic orbits about the collinear points. Celest. Mech. Dyn. Astron. 22(3), 241-253 (1980). doi:10.1007/BF01229511

Salazar, F.J.T., Winter, O.C.: Solar reflectors about the Sun-Earth artificial collinear equilibrium points. In: 67th International Astronautical Congress, International Astronautical Federation, Guadalajara, Mexico (2016)

Salazar, F.J.T., McInnes, C.R., Winter, O.C.: Intervening in Earth's climate system through space-based solar reflectors. Adv. Space Res. 58(1), 17-29 (2016). doi:10.1016/j.asr.2016.04.007

Szebehely, V.: Theory of Orbits: The Restricted Problem of Three Bodies. Academic Press, New York (1967)

Thurman, R., Worfolk, P.: The Geometry of Halo Orbits in the Circular Restricted Three-Body Problem. Technical report GCG95, Geometry Center, University of Minnesota (1996)

Zielinski, G.A., Mayewski, P.A., Meeker, L.D., et al.: Potential atmospheric impact of the Toba Mega-Eruption $\sim 71,000$ years ago. Geophys. Res. Lett. 23(8), 837-840 (1996). doi:10.1029/96GL00706

Zubrin, R., McKay, C.: Technological requirements for terraforming Mars. J. Br. Interplanet. Soc. 50, 83-92 (1997) 